\title{
Structure of Novel Composite Single Yarn from Polypropylene Staple Fibers
}

\author{
Kimura Hirokazu ${ }^{a}$, Matsumoto Masakazu ${ }^{b}$, Matsumoto Yo-ichi ${ }^{c}{ }^{\text {,* }}$, Fukushima Kazunari ${ }^{\text {d }}$ \\ a Technology Research Institute of Osaka Prefecture, 2-7-1 Ayumino, Izumi, Osaka 594-1157, Japan \\ ${ }^{\mathrm{b}}$ College of Industrial Technology, Nihon University, 1-2-1 Izumi-cho, Narashino, Chiba 275-8575, Japan \\ ${ }^{\mathrm{c}}$ Faculty of Textile Science and Technology, Shinshu University, 3-15-6 Tokida, Ueda, Nagano 386-8567, Japan \\ d Daiwabo Holdings Co. LTD., 3-6-8 Kyutaromachi, Chuo-ku, Osaka, Osaka 541-0056, Japan
}

Received 2 April 2012; accepted for publication 8 May 2012

\begin{abstract}
In order to utilize polypropylene staple fiber as textile material and to develop novel spun yarn with good functionality, we investigated how to construct a three-layered structure of composite single yarn and/or adopt the production method of triplet spun yarn using an experimental ring spinning frame. The unique spinning conditions were not only the types of staple fibers but also the arrangement and the distances of three rovings and the twist level of yarn. The following results were obtained: (1) By adopting the production method of triplet spun yarn with three rovings made from the different types of fibers in the arrangement of different rovings under the spinning condition with an equal distance of roving, the yarn combining side-by-side and sheath-core structures could be constructed by one point of yarn formation and one twisting process without the device for controlling of spinning tension; (2) In the spinning method of staple-core spun yarn, it was necessary to control the difference between spinning tensions of the sheath layer with side-by-side structure and the staple-core layer under the spinning condition with the lower twist level of yarn; (3) For constructing the staple-core layer from polypropylene fiber with greater torsional rigidity, it was important not only to control the greater length of drafted fiber strands for the sheath layer but also to choose the fineness and cut length of the sheath and the core fibers and the composition ratio of polypropylene fiber and to have the greater spindle speed in the spinning frame.
\end{abstract}

Key Words: Polypropylene fiber, Three-layered composite single yarn, Staple-core twin spun yarn, Side-by-side structure, Sheath-core structure

\section{Introduction}

In recent years, demands for the characteristics of textile products have been diversifying and intensifying. The characteristics of textile products are varied by combining the properties of fibers and the structures of fiber assemblies; the human body, especially the skin, is very sensitive to their differences. Up until now, although many kinds of fiber materials have been designed and developed, all properties of their fibers cannot be made full use of in textile products. For example, polypropylene fiber has not only the strong points of being lightweight, heat-retentive, and thermoplastic, but also the weak points of poor affinity for dye, thermal expansion, and so on. Because of these weak points, many of its uses are restricted to industrial materials and these strong points cannot be utilized for textile materials. Therefore, it is necessary to combine the composition of fiber materials with the diversification of yarn structures in order to improve the properties of textile products [1-11].

In general, single yarn is produced by one twisting process with one roving on various types of spinning frames and has the simplest structure of spun yarn. Doubled yarn can be made from twisting together two single yarns from the same fibers. Twin spun yarn is produced by one process of twisting together two fiber strands that have been separated in the drafting zone on a modified ring spinning system, resulting in a yarn with the characteristics of a two-fold structure within a conventional single yarn [12-14]. As in the spinning method for twin spun yarn, triplet spun yarn with a three-fold structure is made by supplying three rovings [15]. Therefore, in the one twisting process using the same spinning method, it is possible to make the composition of different fibers and the multilayered side-by-side structure by supplying many rovings. On the other hand, core-spun yarn as typical composite yarn is generally made by combining staple fibers for the sheath

\footnotetext{
*Corresponding author: E-mail: y1matsu@shinshu-u.ac.jp, Tel: +81-268-21-5363, Fax: +81-268-21-5365
} 
layer and a filament yarn for the core layer using a spinning frame, in which the core filament yarn is controlled at an adequate tension by a device. Geometrically speaking, the sheath-core structure of yarn can be constructed by utilizing the difference between spinning tensions and/or path lengths in the inner and outer layers in the yarn. In the spinning method, although it is easy to insert a spun yarn as core yarn, the production of composite yarn needs to have two processes of twisting. In order to decrease labor expenses and reduce the cost of the yarn production, it is necessary to control the tension of the supplied roving or drafted fiber strand in the yarn production with one twisting process. However, although it is very difficult to control the tension of the supplied roving or staple fiber strand in the roller drafting system, it is not difficult to control the spinning tensions of drafted fiber strands which simultaneously emerge from the front roller of the spinning frame. Since the spinning tension of drafted fiber strands can be controlled by strand length and/or distance from the front roller nip to the point of yarn formation, the controlling method has a merit without the tension device $[16,17]$. However, the controlling method has hardly been reported up until now.

Therefore, in order to design and develop novel composite single yarn from polypropylene staple fibers, we investigated how to produce staple-core twin spun yarn and/or combine two spinning methods of core-spun yarn and twin spun yarn into one twisting process. In addition, the effects of the fiber properties and the spinning parameters upon the controlling of the composite yarn structure were examined.

\section{Experiment}

Table 1 lists sample rovings made from various staple fibers. Three kinds of staple fibers with differing fiber fineness and cut length are rayon, acrylic, and polypropylene. Tensile properties of the rovings, especially the relationship between sample length and breaking load were observed using an Instron-type constant rate of elongation tensile tester (TOYO MEASURMENT INSTRUMENTS, TENSILON UTM-III-100) with an extension rate of $500 \mathrm{~mm} / \mathrm{min}$. There were ten tests per roving. Torsional rigidities of fiber strands made from 390 fibers were determined using a torsion tester (KATOTEC, KES-YN1) with a testing length of $15 \mathrm{~mm}$, a tension of $10 \mathrm{cN}$, and ten tests per strand. The experiment room was a controlled environment of $20 \pm 2{ }^{\circ} \mathrm{C}$ and 75 $\pm 5 \%$ R.H.

Fig. 1 shows a schematic illustration for the production method of composite single yarn. Yarn production is an application of the spinning method for triplet spun yarn. Namely, three different types of rovings are supplied into the back roller of a modified ring spinning frame and the drafted fiber strands emerge from the front roller. The supply rovings and/or fiber strands are separated by three mild steel guides located near the back of the back, middle, and front rollers. And then the composite single yarn is produced by one twisting process. The spinning parameters of composite single yarn are a position (and/or arrangement: $\mathbf{A} / \mathbf{B} / \mathbf{C}$ ) of each supply roving made from different fibers, the distance between the center of supply rovings (and/or roving distance: $\boldsymbol{S}$ ), and twist factor $(\boldsymbol{K})$ of yarn. Twist factor of yarn is defined by $K=T w / N e^{1 / 2}=T w /(590.54 /$ $\boldsymbol{T e x})^{1 / 2}$, where $\boldsymbol{T} \boldsymbol{w}$ is twist level of yarn (turns $/ 2.54 \mathrm{~cm}$ ), $\mathbf{N e}$ is English cotton count of yarn (Ne), and $\boldsymbol{T e x}$ is tex count of yarn (tex). Twist level of yarn is defined by $\boldsymbol{T} \boldsymbol{w}=\boldsymbol{R} \boldsymbol{s} / \boldsymbol{R} \boldsymbol{f}$, where $\boldsymbol{R} \boldsymbol{s}$ is spindle speed (rpm), and $\boldsymbol{R} \boldsymbol{f}$ is delivery speed $(2.54 \mathrm{~cm} / \mathrm{min})$ of

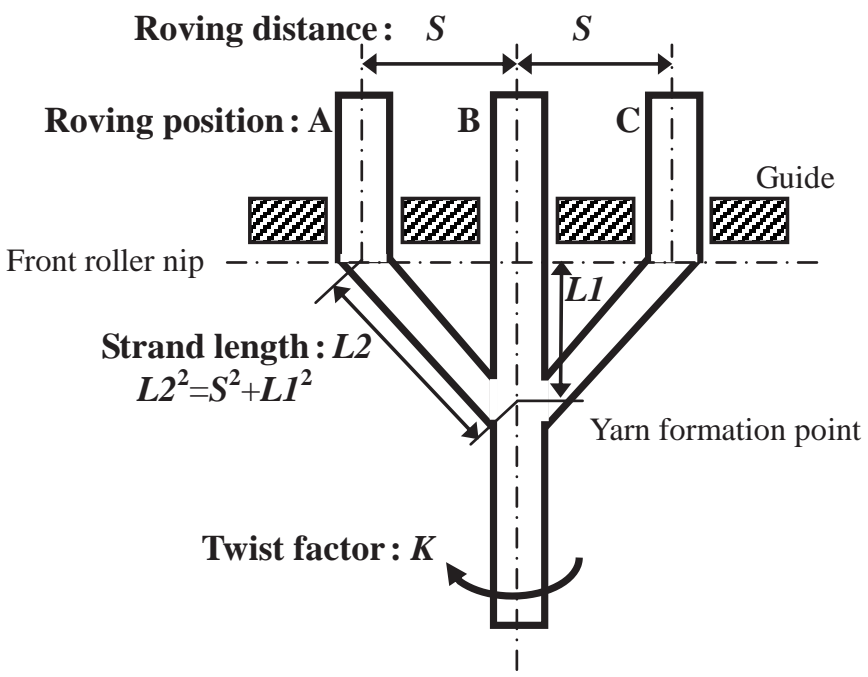

Fig. 1 Schematic illustration of experimental spinning method with three rovings $(\boldsymbol{S}=\boldsymbol{S} \boldsymbol{1}=\boldsymbol{S} 2)$.

Table 1 Sample fibers and rovings

\begin{tabular}{lccccc}
\hline \multicolumn{1}{c}{ Fiber } & $\begin{array}{c}\text { Fineness } \\
(\mathrm{dtex})\end{array}$ & $\begin{array}{c}\text { Cut length } \\
(\mathrm{mm})\end{array}$ & $\begin{array}{c}\text { Roving size } \\
\left(\times 10^{2} \text { tex }\right)\end{array}$ & Specific gravity & $\begin{array}{c}\text { Torsional rigidity } \\
(\mathrm{mg} \cdot \mathrm{cm})\end{array}$ \\
\hline Core & & & & & \\
Polypropylene 1 & 1.7 & 51 & 5.9 & 0.91 & 38 \\
Polypropylene 2 & 1.0 & 38 & 5.9 & 0.91 & 23 \\
Rayon 2 & 1.7 & 51 & 5.9 & 1.50 & 20 \\
Sheath & & & & & 32 \\
Acrylic & 1.7 & 38 & 5.4 & 1.15 & 15 \\
Rayon 1 & 1.4 & 38 & 5.9 & 1.50 & 15 \\
\hline
\end{tabular}


front roller in ring spinning system. In the spinning condition for composite single yarn, the length $(\boldsymbol{L} \boldsymbol{1})$ of the drafted fiber strand located at the center position of $\mathbf{B}$ was measured by a scale in which each measurement was repeated ten times per yarn. The length $(\boldsymbol{L} 2)$ of other strands located at the side position of $\mathbf{A}$ (or $\mathbf{C}$ ) was calculated from the mean value of the center length using the basic trigonometry of $\boldsymbol{L} \mathbf{2}^{2}=\boldsymbol{S}^{\mathbf{2}}+\boldsymbol{L} \boldsymbol{1}^{2}$. The linear density of the yarn produced was about 19.7 tex $(30 \mathrm{Ne})$.

Moreover, yarn with a tension of $3 \mathrm{cN}$ was fixed with a resin (OHKEN SHOJI, BIOLITE) and the cross section of yarn was observed under a microscope (KEYENCE, VHX-500), in which the section was twenty pieces per yarn.

\section{Results and Discussion}

\subsection{Effects of Spinning Parameters}

In applying the spinning method of triplet spun yarn with three rovings, the structure of yarn is greatly influenced by the spinning parameters and the fiber types of roving. And then it is possible to set up two kinds of arrangements; the same rovings and different rovings. In general, triplet spun yarn is made from three of the same ravings. The production of three-layered composite single yarn needs to have three rovings made from different types of fibers in the arrangement of different rovings. Namely, it is provided that the fiber type of roving located at the center position of $\mathbf{B}$ differs from that of roving located at each side position of $\mathbf{A}$ or $\mathbf{C}$, but these two rovings located at both side positions of $\mathbf{A}$ and $\mathbf{C}$ are made from the same fibers.

Fig. 2 shows schematic illustrations for the roving distance, the point of yarn formation, and the cross-sectional views of composite single yarn samples. In adopting the spinning method of triplet spun yarn, it is possible to set up two types of roving distances; $\boldsymbol{S} \boldsymbol{I}=\boldsymbol{S} \mathbf{2}$ and $\boldsymbol{S} \boldsymbol{1}<\boldsymbol{S} \mathbf{2}$ (or $\boldsymbol{S} \boldsymbol{1}>\boldsymbol{S} \mathbf{2}$ ), where $\boldsymbol{S} \boldsymbol{1}$ is the roving distance between the center of $\mathbf{A}$ and $\mathbf{B}$, and $\boldsymbol{S} \mathbf{2}$ is the distance between the center of $\mathbf{B}$ and $\mathbf{C}$. The spinning condition with the same roving distance of $\boldsymbol{S} \boldsymbol{I}$ $=\boldsymbol{S} \mathbf{2}$ has one point of yarn formation. On the other hand, the spinning condition with the different roving distance of $\boldsymbol{S} \boldsymbol{1}<\boldsymbol{S} \boldsymbol{2}$ has two points of yarn formation. Furthermore, the structures of composite single yarn are varied with the spinning tensions of drafted fiber strands emerging from the front roller. Now, it is assumed that each spinning tension of drafted fiber strands located at the position of $\mathbf{A}, \mathbf{B}$, or $\mathbf{C}$ is denoted by $\boldsymbol{T A}, \boldsymbol{T B}$, or $\boldsymbol{T C}$. In the case of one point of yarn formation, if all of the spinning tensions are controlled by $\boldsymbol{T A}=\boldsymbol{T} \boldsymbol{B}=\boldsymbol{T C}$, a composite single yarn has a sideby-side structure. If the spinning tensions are controlled by $\boldsymbol{T B}>\boldsymbol{T A}$ (or $\boldsymbol{T C}$ ) and $\boldsymbol{T A}=\boldsymbol{T C}$, a yarn structure combining side-by-side and sheath-core is constructed. For differentiating these kinds of yarn from each other, the former is called "different fiber of triplet spun yarn" (denoted by "DFTSY") and the latter is called "staple-core twin spun yarn" (denoted by "SCTSY"). On the other hand, in the case of two points of yarn formation, if all of the spinning tensions are controlled by $\boldsymbol{T} \boldsymbol{A}=\boldsymbol{T} \boldsymbol{B}$ and $\boldsymbol{T} \boldsymbol{A}+\boldsymbol{T} \boldsymbol{B}>\boldsymbol{T C}$, the resulting composite single yarn is called "twin staple-core spun yarn" (denoted by "TSCSY"). And if all tensions are controlled by $\boldsymbol{T A}>\boldsymbol{T B}>\boldsymbol{T C}$, the resulting yarn is called "double staple-core spun yarn" (denoted by "DSCSY").

Furthermore, each strand length from the front roller nip to the point of yarn formation is varied with not only the twist level of yarn but also the arrangement and the distance of rovings. Now, it is assumed that each length of drafted strands located at the position of $\mathbf{A}$ (or $\mathbf{C}$ ) or $\mathbf{B}$ is denoted by $\mathbf{L} \mathbf{2}$ or $\boldsymbol{L 1}$. From the basic trigonometry of $\boldsymbol{L} \mathbf{2}^{2}=\boldsymbol{S}^{2}+\boldsymbol{L} \boldsymbol{1}^{2}$, where $\mathrm{S}$ is the same roving distance ( $=\boldsymbol{S} \boldsymbol{1}=\boldsymbol{S} 2$ ), the strand length of $\boldsymbol{L} \boldsymbol{1}$ is smaller than that of $\boldsymbol{L} \mathbf{2}$ and the difference between those of $\boldsymbol{L} \mathbf{1}$ and $\boldsymbol{L} \mathbf{2}$ increases with the increasing of the roving distance $(\boldsymbol{S})$. Therefore, two lengths of drafted fiber strands can be controlled by the roving distance and the twist factor $(\boldsymbol{K})$ of yarn. In the ring spinning frame, although the twist level $(\boldsymbol{T} \boldsymbol{w})$ of yarn increases with the increasing of the spindle speed $(\boldsymbol{R} \boldsymbol{s})$ under a constant condition of delivery speed of the front roller, the formation point of yarn moves up in the direction of front roller and the two lengths ( $\boldsymbol{L} \mathbf{1}$ and $\boldsymbol{L} 2$ ) of drafted fiber strands decrease with the moving of yarn formation point.

\subsection{Effects of Fiber Characteristics}

Fig. 3 shows the relationship between sample length and breaking load of rovings made from various types of fibers. The breaking load of each roving varies with not only the sample length but also the characteristics of fibers. When the sample length is smaller than about $1 / 2$ cut length of fibers in the roving, the breaking load becomes greater. It can be expected that in the cross section of three-layered composite single yarn made from fibers with differing cut length and fineness, the position of each fiber strand mainly depends on the fiber characteristics. It is well known that finer and longer fibers have a tendency to locate at the center of yarn, whereas coarser and shorter fibers have a tendency to be found near the yarn surface [18-20]. Namely, in the supplying of three rovings, when the roving made from fibers with finer fineness, longer cut length, or heavier specific gravity is located at the center position of the roving arrangement, but that with coarser fineness, shorter cut length, or lighter specific gravity is located at the side position of the roving arrangement, the controlling of spinning tension in each drafted fiber strand will become easier. In addition, to construct composite single yarn with a regulated cross section made from different types of staple fibers, it is necessary to control the spinning tension of drafted fiber strands emerging from the front roller of the spinning frame. If all spinning tensions are controlled by $\boldsymbol{T} \boldsymbol{B}>\boldsymbol{T} \boldsymbol{A}$ (or $\boldsymbol{T C}$ ) and $\boldsymbol{T A}=\boldsymbol{T C}$, a yarn combining sideby-side and sheath-core structures is constructed. Namely, it is necessary to control that $\boldsymbol{L} \boldsymbol{1}$ is smaller than about $1 / 2$ cut length of fibers in the strand and each $\boldsymbol{L} \mathbf{2}$ is greater than about $1 / 2$ cut length of fibers in the strand.

Furthermore, the condition of fiber assembly in the cross section 


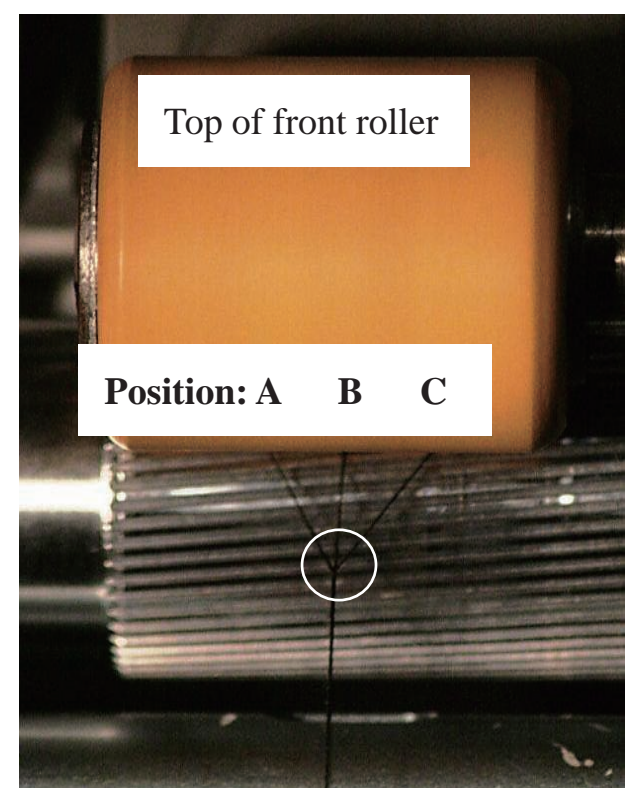

(i) One point of yarn formation ( $\boldsymbol{S} \mathbf{1}=\boldsymbol{S} \mathbf{2}=7 \mathrm{~mm}, \boldsymbol{K}=4.5$, Roving arrangement $(\mathrm{A} / \mathrm{B} / \mathrm{C})=$ rayon $1 /$ rayon $1 /$ rayon 1 )

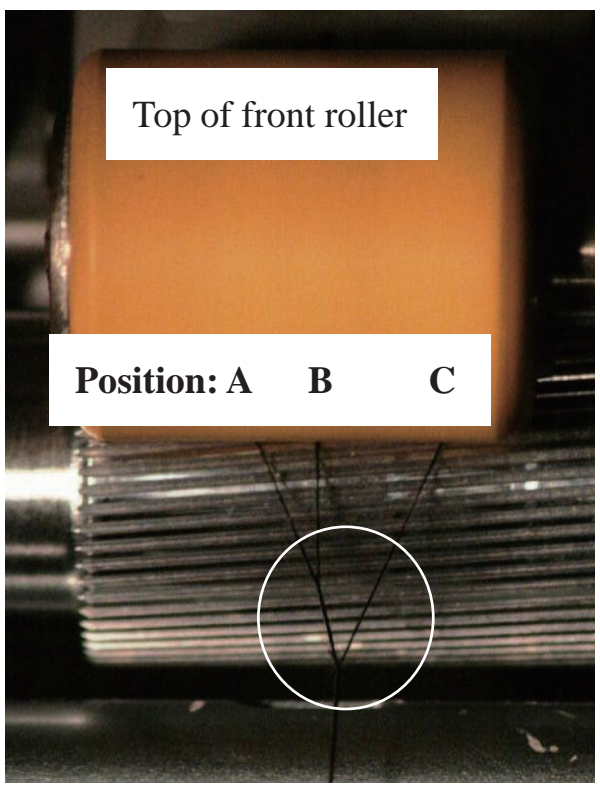

(ii) Two pointsof yarn formation ( $\boldsymbol{S} \mathbf{1}=5 \mathrm{~mm}, \boldsymbol{S} \mathbf{2}=9 \mathrm{~mm}, \boldsymbol{K}=4.5$, Roving arrangement $(\mathrm{A} / \mathrm{B} / \mathrm{C})=$ rayon $1 /$ rayon $1 /$ rayon 1 )

(A-1) Real spinning condition

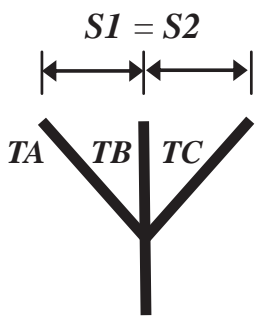

(Roving distance)

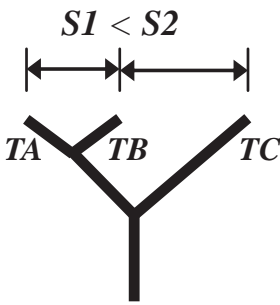

(A-2) Schematic spinning condition

(A) Spinning condition of three drafted fiber strands

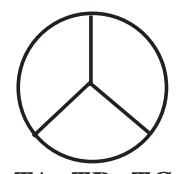

$T A=T B=T C$

(B-1) DFTSY

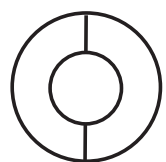

$T B>T A=T C$

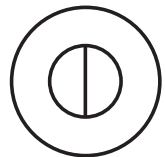

$T A=T B$, $T A+T B>T C$

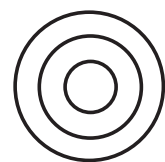

$T A>T B>T C$

\section{(B-2) SCT SY}

(B-3) TSCSY

(B -4) DSCSY

(B) Yarn structure

(i) One point of yarn formation

(ii) Two points of yarn formation

Fig. 2 Relationship between roving distance, yarn formation point, and yarn structure (DFTSY: different fibers of triplet spun yarn, SCTSY: staple core twin spun yarn, TSCSY : twin staple core spun yarn, DSCSY: double staple core spun yarn).

of yarn varies with the torsion property of fiber and the fiber property influences upon the twisting of fiber strands and the propagating of yarn twist. In applying the production method of triplet spun yarn using ring spinning frame, as twisting of yarn is given by rotating of traveler and/or spindle, the twisting of each fiber strand is propagated from the twisting of yarn and the fiber strands have a little level of twist [15].

\subsection{Production and Structure of Composite Single Yarn}

Fig. 4 shows the relationship between conditions and parameters of spinning in the production of composite single yarn, where $\bigcirc$ is a stable condition of continuous spinning, $\triangle$ is an unstable condition of spinning, and $\times$ is an impossible condition of spinning. In this spinning, the fiber composition of core/sheath is 


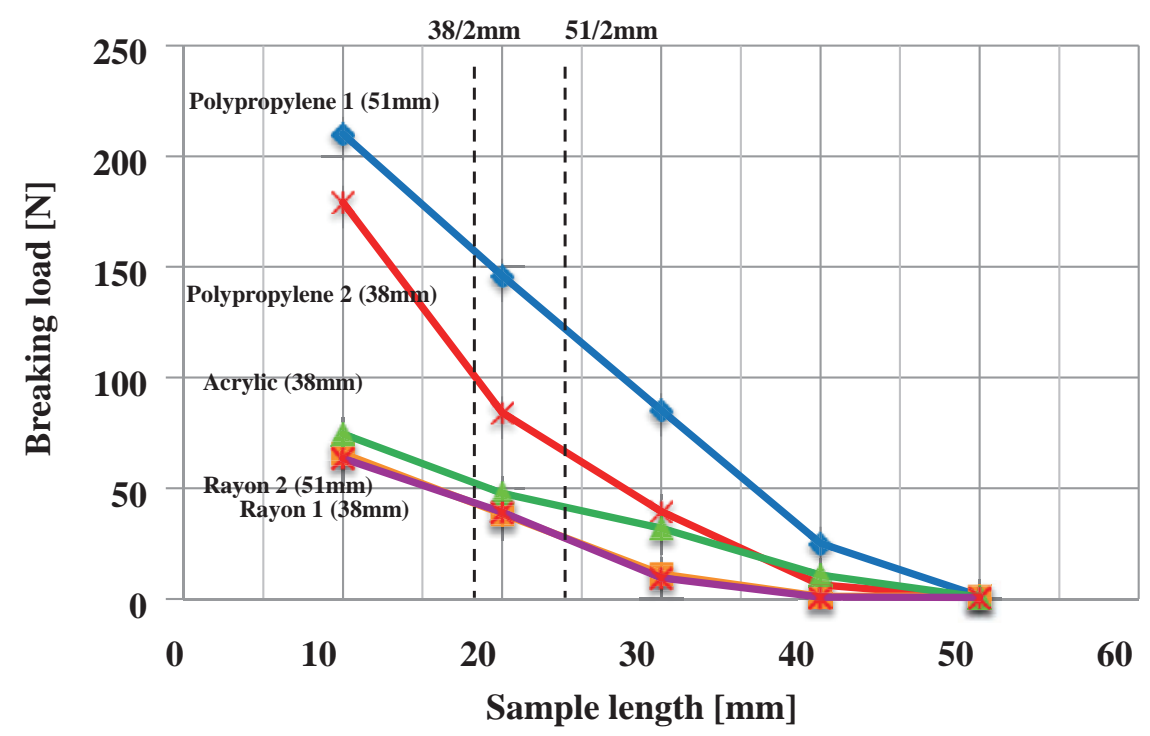

Fig. 3 Relationship between sample length and breaking load of rovings (Each broken line indicates the position at $1 / 2$ cut length of each fiber).
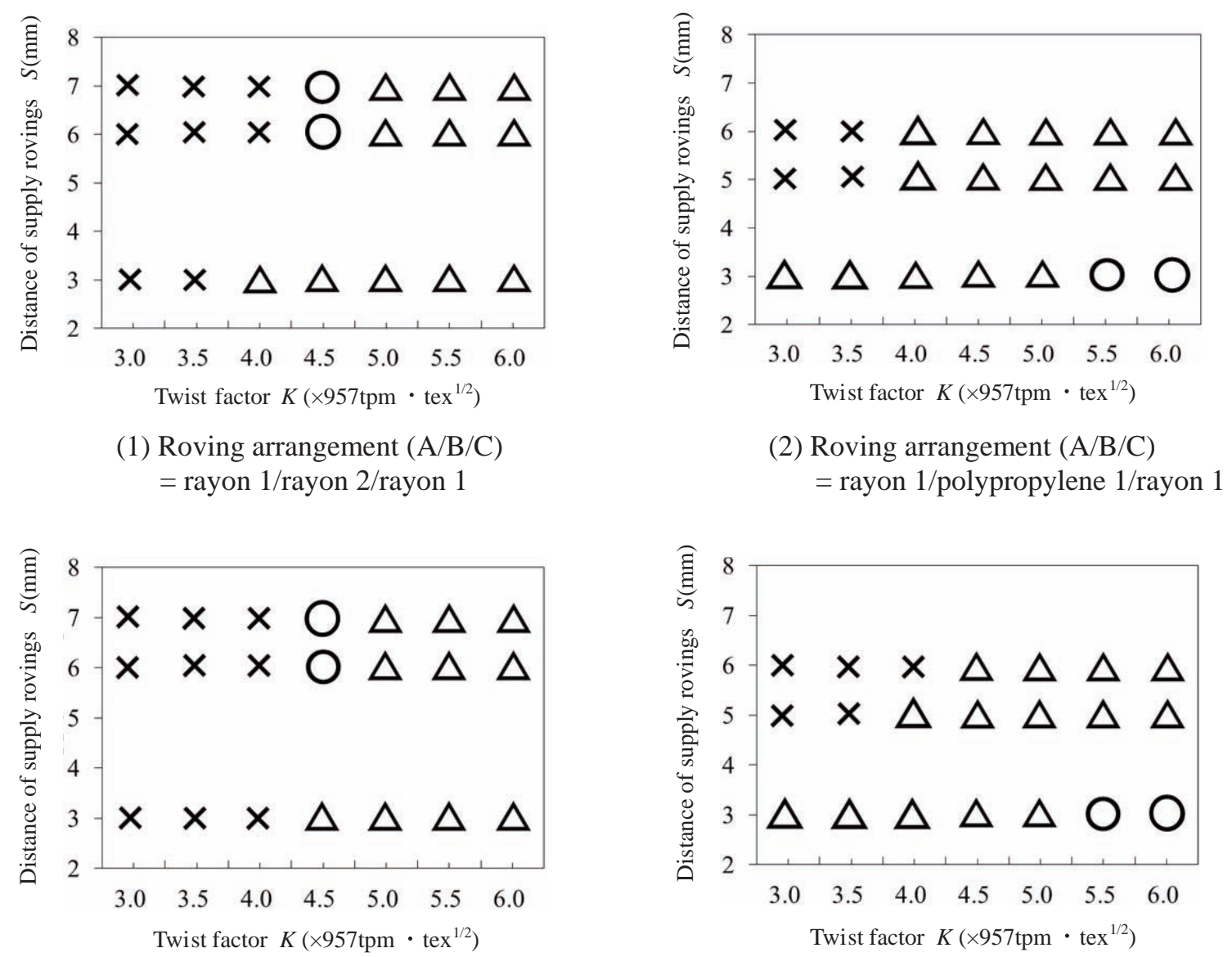

(3) Roving arrangement $(\mathrm{A} / \mathrm{B} / \mathrm{C})$ $=$ acrylic/rayon $2 /$ acrylic

(4) Roving arrangement $(\mathrm{A} / \mathrm{B} / \mathrm{C})$ $=$ acrylic/polypropylene $1 /$ acrylic

Fig. 4 Relationship between spinning condition, twist factor, and roving distance $(\boldsymbol{S}=\boldsymbol{S} \boldsymbol{1}=\boldsymbol{S} \boldsymbol{2}$, $\bigcirc$ : Stable spinning condition, $\triangle$ : Unstable spinning condition, $\times$ : Impossible spinning, Fiber composition of core $/$ sheath $=35 / 65 \%$, Spindle speed $(\boldsymbol{R} \boldsymbol{s})=9,000 \mathrm{rpm}$, Traveler weight $(\boldsymbol{T r})=0.18 \mathrm{cN})$. 
REAL

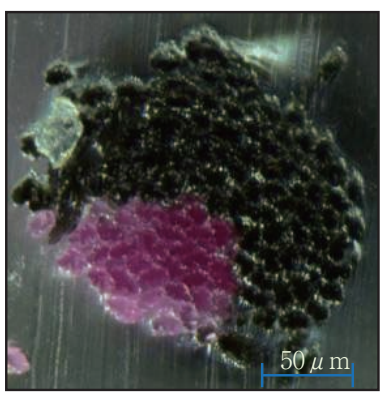

(1) Roving arrangement $(\mathrm{A} / \mathrm{B} / \mathrm{C})$ $=$ rayon $1 /$ rayon $2 /$ rayon $1(\boldsymbol{S}=\boldsymbol{S I}$ $=\boldsymbol{S} \mathbf{2}=6 \mathrm{~mm}, \boldsymbol{K}=4.5, \boldsymbol{L} \boldsymbol{1}=21.0$ $\mathrm{mm}<51 / 2, \boldsymbol{L} \boldsymbol{2}=21.8 \mathrm{~mm}>38 / 2$ )

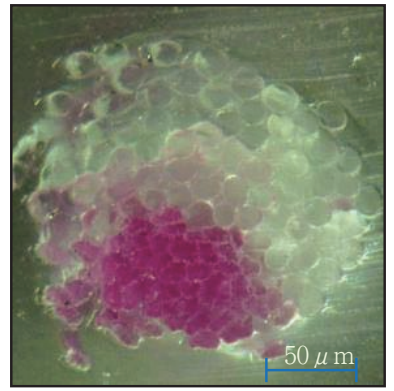

(3) Roving arrangement $(\mathrm{A} / \mathrm{B} / \mathrm{C})$ $=$ acrylic/rayon $2 /$ acrylic $(\boldsymbol{S}=\boldsymbol{S} \boldsymbol{I}$ $=\boldsymbol{S} \mathbf{2}=7 \mathrm{~mm}, \boldsymbol{K}=4.5, \boldsymbol{L} \boldsymbol{I}=20.5$ $\mathrm{mm}<51 / 2, \boldsymbol{L} 2=21.6 \mathrm{~mm}>38 / 2$ )
REAL
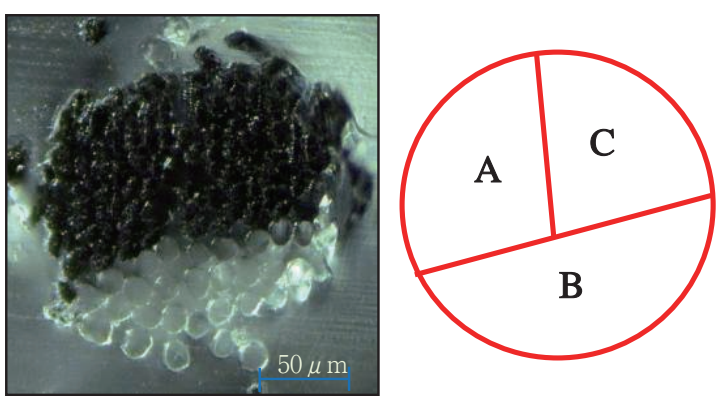

(2) Roving arrangement $(\mathrm{A} / \mathrm{B} / \mathrm{C})$ $=$ rayon $1 /$ polypropylene $1 /$ rayon 1 $(\boldsymbol{S}=\boldsymbol{S} \boldsymbol{I}=\boldsymbol{S} \mathbf{2}=3 \mathrm{~mm}, \boldsymbol{K}=6.0, \quad \boldsymbol{L} \boldsymbol{1}=$ $10.1 \mathrm{~mm}<51 / 2, \boldsymbol{L} 2=10.5 \mathrm{~mm}<38 / 2$ )
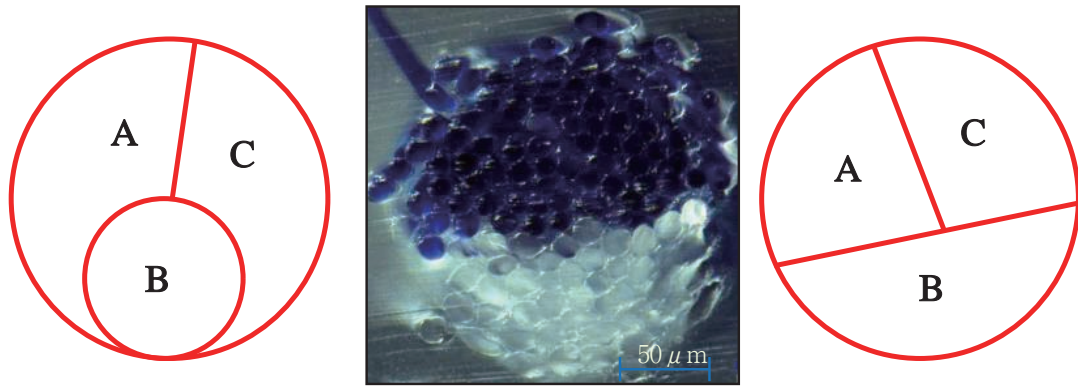

(4) Roving arrangement $(\mathrm{A} / \mathrm{B} / \mathrm{C})$ $=$ acrylic/polypropylene $1 /$ acrylic $(\boldsymbol{S}=\boldsymbol{S} \boldsymbol{1}=\boldsymbol{S} \mathbf{2}=3 \mathrm{~mm}, \boldsymbol{K}=\mathbf{6} .0, \boldsymbol{L} \boldsymbol{1}=$ $9.3 \mathrm{~mm}<51 / 2, \boldsymbol{L} 2=9.7 \mathrm{~mm}<38 / 2$ )

Fig. 5 Typical cross-sectional views of yarn samples (Fiber composition of core $/$ sheath $=35 / 65 \%, \boldsymbol{R s}=9,000 \mathrm{rpm}, \boldsymbol{T r}=0.18 \mathrm{cN}$ ).

$35 / 65 \%$, the spindle speed $(\boldsymbol{R s})$ is $9,000 \mathrm{rpm}$, and the traveler weight $(\boldsymbol{T r})$ is $0.18 \mathrm{cN}$. By supplying three rovings from different types of fibers, the conditions and parameters of spinning were greatly varied with the properties of fiber located at the center position of $\mathbf{B}$. The stable spinning condition was especially affected by the torsional rigidities of the fibers, but the types of fibers in the strands located at the side positions of $\mathbf{A}$ and $\mathbf{C}$ had hardly any influence. Namely, in a comparison with the strand from rayon2 fibers, that from polypropylene1 fibers with greater torsional rigidity needed to have the spinning parameters with a smaller distance of roving and a greater twist factor of yarn. And, in the case of the same fineness of fibers, the diameter of polypropylene fiber with smaller specific gravity was greater than that of rayon fiber.

Fig. 5 shows typical cross sectional views of composite single yarn made under the stable spinning condition. The schematic illustration with red lines was constructed from observation of border lines between three strands of $\mathbf{A}, \mathbf{B}$, and $\mathbf{C}$. In all cross sections of yarns, three-layered structures made from different types of fibers were constructed. However, in the production of staple-core twin spun yarn, although the side-by-side structure for making the sheath layer was constructed by two drafted fiber strands located at the side positions of $\mathbf{A}$ and $\mathbf{C}$, the fiber assembled condition of staple-core layer from the drafted fiber strand located at the center position of $\mathbf{B}$ varied with the fiber properties and the spinning condition. Furthermore, although the fiber assemblies for the staple-core layer were located near the yarn surface, the fiber assembly from rayon 2 fibers had a circular assembled condition but that from polypropylene 1 fibers had a greater extended condition in the direction of yarn diameter. For constructing the staple-core layer in a circular shape, the drafted strand from polypropylene fibers with greater torsional rigidity has to have a higher level of twist. However, in the spinning condition with the higher level of twist, as each fiber strand for the sheath layer also has the higher level of twist, the circular fiber assemblies will be made up under a greater spinning tension. And then, even though the yarn production has an optimal difference between the spinning tensions of sheath and core layers, it can be expected that the circular sheath layer has a lack of width for covering of the staple-core layer. Conversely, for covering the staple-core layer with the sheath layer of side-by-side structure, it is necessary that each drafted strand located at the side positions has a greater width under the spinning 

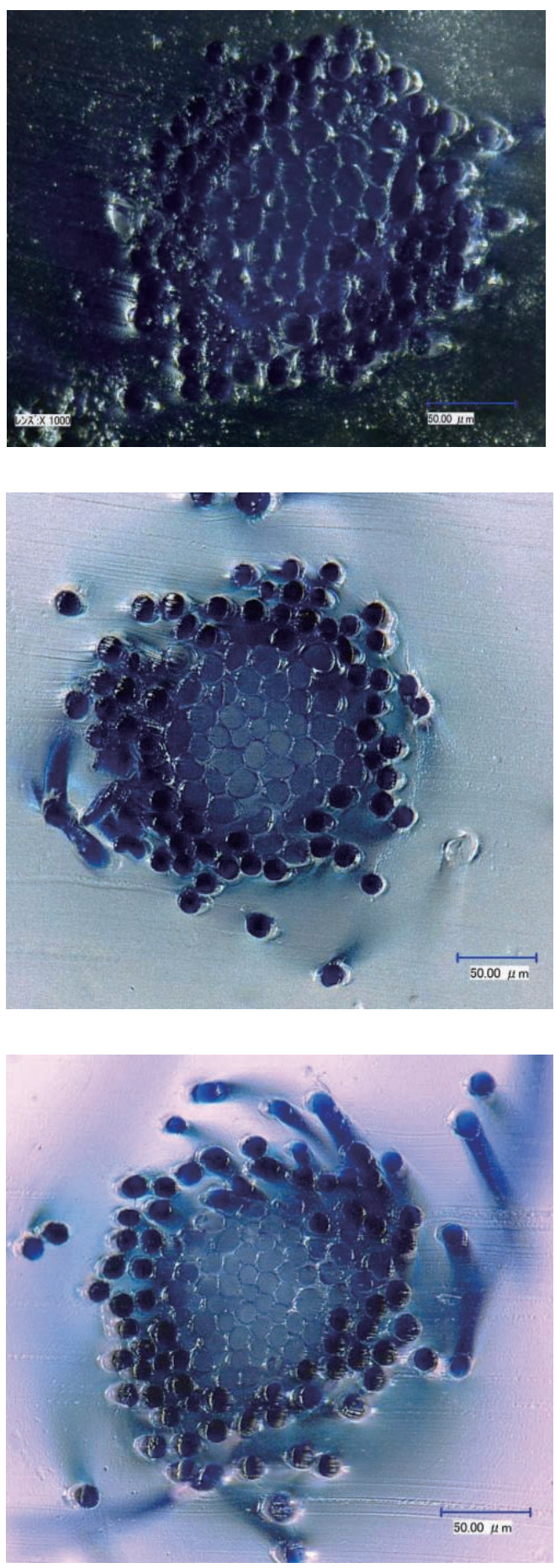

(a) Roving arrangement $(\mathrm{A} / \mathrm{B} / \mathrm{C})=$ acrylic/polypropylene 1/acrylic, $\boldsymbol{S}=\boldsymbol{S} \mathbf{1}=\boldsymbol{S} \mathbf{2}=3 \mathrm{~mm}, \boldsymbol{K}=3.0, \boldsymbol{L} \mathbf{I}=$ $15.1 \mathrm{~mm}<51 / 2, \boldsymbol{L} 2=15.4 \mathrm{~mm}<38 / 2$, Fiber composition of core/sheath $=$ $30 / 70 \%, \boldsymbol{R} \boldsymbol{s}=9,000 \mathrm{rpm}, \boldsymbol{T r}=0.13 \mathrm{cN}$

(b) Roving arrangement $(\mathrm{A} / \mathrm{B} / \mathrm{C})=$ acrylic/polypropylene1/acrylic, $\boldsymbol{S}=\boldsymbol{S} \boldsymbol{1}=\boldsymbol{S} \mathbf{2}=9 \mathrm{~mm}, \boldsymbol{K}=3.0, \boldsymbol{L} \mathbf{1}$ $29.4 \mathrm{~mm}>51 / 2, \boldsymbol{L} 2=30.7 \mathrm{~mm}$ $>38 / 2$, Fiber composition of core $/$ sheath $=35 / 65 \%, \boldsymbol{R s}=$ $11,000 \mathrm{rpm}, \boldsymbol{T r}=0.13 \mathrm{cN}$

(c) Roving arrangement $(\mathrm{A} / \mathrm{B} / \mathrm{C})=$ acrylic/polypropylene 2/acrylic, $\boldsymbol{S}=\boldsymbol{S} \mathbf{1}=\boldsymbol{S} \mathbf{2}=9 \mathrm{~mm}, \boldsymbol{K}=3.0, \boldsymbol{L} \mathbf{1}=$ $28.7 \mathrm{~mm}>38 / 2, \boldsymbol{L} 2=30.0 \mathrm{~mm}$ $>38 / 2$, Fiber composition of core $/$ sheath $=35 / 65 \%, \boldsymbol{R s}=$ $11,000 \mathrm{rpm}, \boldsymbol{T r}=0.13 \mathrm{cN}$

Fig. 6 Typical cross-sectional views of staple core twin spun yarn.

condition with a lower level of twist. Namely, similar to the staplecore layer from rayon2 fibers, even though that from polypropylene1 fibers has a little level of twist propagated from twisting of yarn, the circular condition of staple-core layer will be constructed by transverse force generated from twisting and covering with the sheath layer of side-by-side structure. Therefore, in the production of staple-core twin spun yarn, the most important control is the difference between the spinning tensions of staplecore and sheath layers in the spinning condition with the lower twist level of yarn. However, the spinning system has many limitations in controlling the spinning parameters: the width of the top front roller $(21 \mathrm{~mm})$ and the gauge of the strand guide $(2 \sim 8 \mathrm{~mm}$ with division of $1 \mathrm{~mm}$ ) for the distance between drafted fiber strands, the twist factor of yarn $\left(3.0 \sim 6.0 \times 957 \mathrm{tpm} \cdot \operatorname{tex}^{1 / 2}\right)$, and so on. Then, within the range of spinning parameters in the system, it was very difficult to control an optimal difference between the spinning tensions of drafted fiber strands and construct the staple-core layer with the twin sheath layers.

Therefore, for improving the fiber assembled conditions in the cross section of yarn, it is necessary that the sheath layer from 
fibers with a coarser fineness or a shorter cut length have a longer length of drafted fiber strand in the spinning and that the staplecore layer is made from polypropylene fibers with a finer fineness, a shorter cut length, or a lower composition rate of yarn. In addition, it was necessary to control the spinning tension and/or the length of drafted fiber strand by means of other parameters for the spinning: spindle speed, traveler weight, and so on.

Fig. 6 shows typical cross sections of the staple-core twin spun yarn produced. In a comparison with Fig. 5, the cross sections of sample kinds of yarn had circular assembled conditions of the staple-core layer from polypropylene fibers and an optimal covering of the sheath layer with the side-by-side structure. By decreasing the fineness, cut length, and composition ratio of polypropylene fibers and/or increasing the spindle speed, namely fiber fineness of $1.0 \mathrm{dtex}$, cut length of $38 \mathrm{~mm}$, composition ratio of $30 \%$, spindle speed (Rs) of 11,000 rpm and/or traveler weight $(\boldsymbol{T r})$ of $0.13 \mathrm{cN}$, it can be possible to construct the optimal difference between spinning tensions for the staple-core and the sheath layers, the circular assembled condition for the staple-core layer, and the sheath layer with the lower level of twist. However, although the spinning tension increased with the increasing of a traveler weight as spinning parameter, the lengths of drafted strands hardly varied with the increasing of the traveler weight. Therefore, in the production of staple-core twin spun yarn from polypropylene fibers, it was necessary to consider not only the characteristics of fibers for the staple-core layer but also the spindle speed of the ring spinning frame for controlling the spinning tension of drafted fiber strand. Namely, in the case of polypropylene fibers with greater spinning tension, although it was not important to control that $\boldsymbol{L} \boldsymbol{1}$ was smaller than about $1 / 2$ cut length of fibers in the strand, it was very important to control that each $\boldsymbol{L} \mathbf{2}$ was significantly greater than about $1 / 2$ cut length of fibers in the strand.

\section{Conclusions}

In order to utilize polypropylene staple fiber as textile material and to develop novel composite single yarn with good functionality, we investigated how to produce staple-core twin spun yarn by combining side-by-side and sheath-core structures into a yarn. The following results were obtained: (1) By applying the production method of triplet spun yarn on the experimental ring spinning system, the yarn combining side-by-side and sheath-core structures could be constructed by one point of yarn formation and one twisting process without the device for controlling of spinning tension; (2) In the spinning method of staple-core spun yarn, it was necessary to control the difference between spinning tensions of the sheath layer with side-by-side structure and the staple-core layer under the spinning condition with the lower twist level of yarn; (3) For constructing the staple-core layer from polypropylene fibers with greater torsional rigidity, it was important not only to control the greater length of drafted fiber strands for the sheath layer but also to choose the fineness and cut length of the sheath and the staple-core fibers and the composition ratio of polypropylene fiber and to increase the spindle speed of the spinning frame.

\section{Acknowledgments}

We wish to thank Mr. FUKAGAWA Naoya, a graduate student of Shinshu University, for his assistance.

\section{References}

[1] Matsumoto Y, Toriumi K, Tsuchiya I, Harakawa K (1992) Text Res J, 62, 710-714.

[2] Wu W, Lee J (1995) Text Res J, 65, 225-229.

[3] Matsumoto Y, Sakaguchi A, Toriumi K, Morooka H, Harakawa K (1997) J Seric Sci Jpn, 66, 6-10.

[4] Cheng K B, Murray R (2000) Text Res J, 70, 690-695.

[5] Matsumoto Y, Fushimi S, Saito H, Sakaguchi A, Toriumi K, Nishimatsu T, Shimizu Y, Shirai H, Morooka H, Gong H (2002) Text Res J, 72, 735-740.

[6] Soe A, Takahashi M, Nakajima M, Matsuo T, Matsumoto T (2004) Text Res J, 74, 819-826.

[7] Matsumoto Y, Saito H, Sakaguchi A, Toriumi K, Nishimatsu T, Shimizu Y, Shirai H, Morooka H, Gong H (2004) Text Res J, 74, 671-676.

[8] Fatma G, Demet Y, Özer G (2006) Text Res J, 76, 226-234.

[9] Su C, Fang J (2006) Text Res J, 76, 441-447.

[10] Basal G, Oxenham W (2007) Text Res J, 76, 567-575.

[11] Kimura H, Morishima M, Nishioka T, Wakako L, Matsumoto Y (2009) J Text Eng, 55, 187-192.

[12] Gore C (1982) Text Ind, 146, 64-65.

[13] Happey F (1982) “Contemporary Textile Engineering”, p.87, Academic Press, London.

[14] Matsumoto Y, Tsuchiya I, Toriumi K, Harakawa K (1991) Text Res J, 61, 131-136.

[15] Matsumoto Y, Saito H, Toriumi K, Morooka H, Harakawa K (1997) J Seric Sci Jpn, 66, 323-329.

[16] Matsumoto Y, Kimura H, Yamamoto T, Matsuoka T, Fukushima K (2009) Text Res J, 79, 947-952.

[17] Matsumoto Y, Kanai H, Morooka H, Kimura H, Fukushima K (2010) Text Res J, 80, 1056-1064.

[18] Boswell H R, Townend P P (1955) J Text Inst, 46, T778-779.

[19] Morton W E (1956) J Text Inst, 47, T239-240.

[20] Balasubramanian N (1970) Text Res J, 40, 129-141. 